\title{
Physics case for a polarised target for AFTER@LHC
}

\author{
J.P. Lansberg ${ }^{* 1}$, M. Anselmino ${ }^{2}$, R. Arnaldi ${ }^{2}$, S.J. Brodsky ${ }^{3}$, V. Chambert ${ }^{1}$, \\ J.P. Didelez ${ }^{1}$, M.G Echevarria ${ }^{4}$, E.G. Ferreiro ${ }^{5}$, F. Fleuret ${ }^{6}$, Y. Gao ${ }^{7}$, B. Genolini ${ }^{1}$, \\ C. Hadjidakis ${ }^{1}$, I. Hřivnáčová ${ }^{1}$, D. Kikola ${ }^{8}$, A. Kurepin ${ }^{9}$, C. Lorcé ${ }^{10}$, L. Massacrier ${ }^{1,11}$, \\ A. Nass ${ }^{12}$, C. Pisano ${ }^{13}$ I. Schienbein ${ }^{14}$, M. Schlegel ${ }^{15}$, E. Scomparin ${ }^{2}$, J. Seixas ${ }^{16}$, \\ A. Signori ${ }^{17}$, E. Steffens ${ }^{18}$, N. Topilskaya ${ }^{9}$, B. Trzeciak $^{19}$ U.I. Uggerhøj $^{20}$, A. Uras ${ }^{21}$, \\ R. Ulrich ${ }^{22}$, and Z. Yang ${ }^{6}$.
}

${ }^{1}$ IPNO, Univ. Paris-Sud, CNRS/IN2P3, Université Paris-Saclay, Orsay, France

${ }^{2}$ Dip. di Fisica and INFN Sez. Torino, Via P. Giuria 1, Torino, Italy

${ }^{3}$ SLAC National Accelerator Laboratory, Stanford University, Menlo Park, USA

${ }^{4}$ ECM, Universitat de Barcelona, Barcelona, Spain

${ }^{5}$ Dept. de Física de Partículas, USC, Santiago de Compostella, Spain

${ }^{6}$ LLR, École Polytechnique, CNRS/IN2P3, Palaiseau, France

${ }^{7}$ CHEP, Department of Engineering Physics, Tsinghua University, Beijing, China

${ }^{8}$ Faculty of Physics, Warsaw University of Technology, Warsaw, Poland

${ }^{9}$ Institute for Nuclear Research, Russian Academy of Sciences, Moscow, Russia

${ }^{10}$ CPhT, Ecole Polytechnique, CNRS, Université Paris-Saclay, Palaiseau, France

${ }^{11}$ LAL, Univ. Paris-Sud, CNRS/IN2P3, Université Paris-Saclay, Orsay, France

${ }^{12}$ Institut für Kernphysik, Forschungszentrum Jülich, Jülich, Germany

${ }^{13}$ Dipartimento di Fisica, Universita degli Studi di Pavia, Pavia, Italy

${ }^{14}$ LPSC, Univ. Joseph Fourier, CNRS/IN2P3/INPG, Grenoble, France

15 Institute for Theoretical Physics, Tübingen U., Tübingen, Germany

${ }^{16}$ LIP and IST, Lisbon, Portugal

${ }^{17}$ Nikhef and Dept. of Physics and Astronomy, VU Amsterdam, Amsterdam, The Netherlands

${ }^{18}$ Physics Institute, Friedrich-Alexander University Erlangen-Nr̈nberg, Erlangen, Germany

${ }^{19}$ Institute for Subatomic Physics, Utrecht University, Utrecht, The Netherlands

${ }^{20}$ Department of Physics and Astronomy, University of Aarhus, Denmark

${ }^{21}$ IPNL, Université Claude Bernard Lyon-I, CNRS/IN2P3, Villeurbanne, France

${ }^{22}$ Institut für Kernphysik, Karlsruhe Institute of Technology (KIT), Karlsruhe, German

\begin{abstract}
We review a number of ideas put forward in favour of the use of a polarised target along with the proposed idea of a fixed-target experiment using the LHC beams - AFTER@LHC. A number of recent studies have shown that single transverse-spin asymmetries (STSAs) are large enough to be precisely measured in the region accessible with AFTER@LHC, in particular as regards the Drell-Yan process as well as single-pion, isolated-photon and jet production. AFTER@LHC with a polarised target would also be the ideal experimental set-up to measure the gluon Sivers effect via a number of original quarkonium STSA studies. We discuss first figures-of-merit based on simulations for AFTER@LHC with a polarised target.
\end{abstract}

XVIth International Workshop in Polarized Sources, Targets, and Polarimetry, PSTP2015,

14-18 September 2015,

Bochum, Germany 


\section{Introduction}

To date, RHIC is the only collider of polarised protons which allows to investigate spin physics at ultra relativistic energies, well beyond the mass of most hadronic resonances, and in the production of weak bosons. Such measurements were not possible at the Fermilab Tevatron nor are at the LHC since their beams are not polarised. It is however possible to use a polarised target impinged by the LHC beams to perform new and unique investigations of single transverse spin asymmetries (STSAs), $A_{N}$. These are related to the Sivers effect [1] observed since long ago whereby the transverse momentum of the partons, $k_{T}$, is correlated to the nucleon spin. Such a dynamics is normally encoded in Transverse-Momentum-Dependent distributions (referred to as TMDs in the following) when $k_{T}$ is explicit or to twist-3 correlators, such as $T_{F}(x, x)$ which is also known as the Efremov-Teryaev-Qiu-Sterman quark-gluon correlator.

In the AFTER@LHC case, owing to the boost between the laboratory and the centre-of-mass frames in the fixed-target mode, measurements of STSAs in the rapidity region covered by experiments such as LHCb and ALICE would probe the region of large parton momentum fractions in the polarised hadron, $x^{\uparrow}[2,3,4]$. This is precisely where the asymmetries are expected to be the largest as recently quantified for Drell-Yan pair production (DY) [5, 6], isolated photon [6,7], single pion $[6,7]$ or jet [7] production. These STSAs, which are attributed to the dynamics of the quarks inside the polarised hadrons, are thus directly (or indirectly, depending on the process) related to the quark Sivers effect. In the DY case, the extracted quark Sivers function is predicted $[8,9]$ to have an opposite sign w.r.t. to the Semi-Inclusive-Deep-Inelastic-Scattering case. The verification of this predicted sign change is the main physics case of the DY COMPASS run [10] and the experiments E1039 [11] and E1027 [12] at Fermilab, with a polarised target (resp. beam) to investigate lower (resp. larger) $x^{\uparrow}$. AFTER @LHC is an extremely complementary facility to further investigate the quark Sivers effect by measuring DY STSAs [5, 6] over a wide range of $x \uparrow$ in a single set-up.

As regards the gluon Sivers effect, nearly nothing is known and most of the observational constraints are weak, if not model-dependent. The reader is guided to [13] for a recent overview. Yet, the PHENIX collaboration was the first to look at STSAs of gluon sensitive probes with that of $J / \psi$ production [14]. They found out an $A_{N}$ compatible with zero but also allowing for values as large as $20 \%$. In addition, such an $A_{N}$ is not directly connected to the gluon Sivers function since $k_{T}$ is integrated over in $A_{N}$ and is thus more naturally connected to twist- 3 correlators (see also [15]). With the large luminosities offered by AFTER@LHC, similar STSAs for bottomonia, spin-singlet states such as the $\eta_{c}$ [16] or associated production [3] become accessible paving the way for a convincing program to measure the gluon Sivers effect in order to assess the importance of the gluon orbital angular momentum in the resolution of the nucleon-spin puzzle.

\section{Two approaches for a fixed-target experiment at the LHC with a polarised target}

We briefly discuss here two promising options allowing the highly energetic LHC beams to collide onto a target. Both are currently investigated in the LHC complex. The first option relies on the use of a bent crystal which is positioned in the halo of the LHC beam [17]. A few protons

\footnotetext{
*Speaker.
} 
(or $\mathrm{Pb}$ ions) per bunch per pass can then be channelled along the lattice of the crystal. Since it is bent, these particles are deflected by a few mrad w.r.t the beam axis along the crystal curvature. This extraction technique is efficient and it allows one to obtain a clean and well collimated beam. A remarkable asset of this technique is that it does not affect the LHC performances since, by essence, it only uses beam particles which would anyway be lost for collisions. Such a technique, used in a similar manner, is in fact currently investigated as a possible means to improve the LHC beam collimation by the (L)UA9 collaboration [18]: two goniometres and two bent crystals has so far been installed in the LHC beam pipe at IR7 and tested with success [19]. This follows other successful data takings for protons at CERN (SPS) [20], Fermilab [21], Protvino [22] and for lead ions at CERN SPS [23].

The second option relies on the use of an internal gas target which can be polarised, unlike an internal wire target as used at DESY by Hera-B [24] and discussed for the LHC in [25]. The low density of the gas also allows for a parasitic functioning where the beam lifetime is essentially not affected, yet with very large luminosities (see below). Such an internal gas-target option is in fact currently used by the LHCb collaboration but as a luminosity monitor [26] (SMOG) initially designed to monitor the transverse size of the beam. Even with a limited pressure (about $10^{-7}$ mbar), it demonstrates the viability of such a solution over extended periods of time, without any interferences on the other LHC experiments. LHCb performed pilot runs with $p$ and $\mathrm{Pb}$ beams on a Ne gas target in 2012 and 2013. With the corresponding beam energies, the c.m.s energy was $\sqrt{s_{N N}}=86.6 \mathrm{GeV}$ for $p$ and $54.5 \mathrm{GeV}$ for $\mathrm{Pb}$. The success of such pilot runs motivated longer runs in 2015: $p$ on $\mathrm{Ne}$ (12 hours), $\mathrm{He}$ (8 hours) and $\mathrm{Ar}$ (3 days) at $110.4 \mathrm{GeV}$ as well as $\mathrm{Pb}$ on $\mathrm{Ar}(1$ week) and $p$ on $\operatorname{Ar}$ (a few hours) at $68.6 \mathrm{GeV}$. No decrease of the LHC performances was observed. We stress that the current gas pressure is limited by the pumping system. Using a system like the one of the HERMES experiment at DESY-HERA [27], higher pressures can be obtained with the further possibility of polarised gases [28].

With the bent-crystal option, an average of 15 protons each 25 ns should be extracted. This means a flux of $5 \times 10^{8} p^{+} \mathrm{s}^{-1}$ entirely from the LHC beam losses ${ }^{1}$. In Tab. 1, we gathered the instantaneous and per annum luminosities (assuming a run of $10^{7} \mathrm{~s}$ for $p^{+}$over a year) for $p^{+}$on $\mathrm{H}$ and $\mathrm{D}$ targets. One obtains integrated luminosities as large as $20 \mathrm{fb}^{-1}$ with a $1 \mathrm{~m}$-long target of liquid hydrogen; such a number is comparable to the data sample collected at 7 and $8 \mathrm{TeV}$ at the collider LHC.

With the internal gas-target option, the luminosity is given by the product of the particle current $I$ and the areal density of the polarised storage cell target, $\theta$. The density is affected by many parameters, the most important being the flux of the polarised source injected into the target cell and the geometry of the cell [31]. For the LHC geometry [31] and assuming the flux from the HERMES target source, a density of $2.5 \times 10^{14} \mathrm{~cm}^{-2}$ for a $1 \mathrm{~m}$ long cell for $\mathrm{H}$ at $300 \mathrm{~K}$ can be expected. Such a target density is far below the densities affecting the proton beam life time. In the case of the $p^{+}$beam, $I=3.14 \times 10^{18} p^{+} \mathrm{s}^{-1}$. The resulting luminosity is shown in Table $1 \mathrm{~b}$. The storage cell target can be used as well with unpolarised gas of larger atomic masses with the LHC lead beams to study heavy-ion collisions at $72 \mathrm{GeV}$. The limit of such a solution is essentially set

\footnotetext{
${ }^{1}$ We note that contrary to some statements made in the 90 's, the degradation of the crystal is negligible as shown in [29]. It should be at the level of $6 \%$ per $10^{20}$ particles $/ \mathrm{cm}^{2}$, i.e. 1 year of operation. The crystal can simply be moved by less than a millimeter or replaced after a year such that the beam halo hits an intact spot.
} 


\begin{tabular}{|c|c|c|c|c|c|c|}
\hline Beam & Target & $\begin{array}{l}\text { Thickness } \\
(\mathrm{cm})\end{array}$ & $\begin{array}{c}\rho \\
(\text { g.cm } \\
-3)\end{array}$ & A & $\begin{array}{c}\mathscr{L} \\
\left(\mu \mathrm{b}^{-1} \cdot \mathrm{s}^{-1}\right)\end{array}$ & $\begin{array}{c}\int \mathscr{L} \\
\left(\mathrm{fb}^{-1} \cdot \mathrm{y}^{-1}\right)\end{array}$ \\
\hline$p$ & Liquid $\mathrm{H}$ & 100 & 0.068 & 1 & 2000 & 20 \\
\hline$p$ & Liquid D & 100 & 0.16 & 2 & 2400 & 24 \\
\hline
\end{tabular}

(a) Bent-crystal option

\begin{tabular}{cccccc} 
Beam & Target & $A$ & $\begin{array}{c}\text { Areal density }(\theta) \\
\left(\mathrm{cm}^{-2}\right)\end{array}$ & $\begin{array}{c}\mathscr{L} \\
\left(\mu \mathrm{b}^{-1} \cdot \mathrm{s}^{-1}\right)\end{array}$ & $\begin{array}{c}\int \mathscr{L} \\
\left(\mathrm{fb}^{-1} \cdot \mathrm{y}^{-1}\right)\end{array}$ \\
\hline$p$ & $\mathrm{H}$ & 1 & $2.5 \times 10^{14}$ & 900 & 9 \\
$p$ & $\mathrm{D}$ & 2 & $3.2 \times 10^{14}$ & 1200 & 12
\end{tabular}

(b) Polarised internal-gas-target option

Table 1: Expected luminosities (a) with a $7 \mathrm{TeV}$ proton beam extracted by means of bent crystal on conventional targets and (b) with an internal gas-target inspired by the HERMES experiment.

by the number of beam particles "consumed" by the target over a fill or the data acquisition of the detector. We have also gathered the instantaneous and yearly luminosities with such a system in Tab. 1b.

As what regards the expectations for the target polarisation, the case of the gas target is simple. By reusing a target like that of HERMES, the effective polarisation of $\mathrm{H}$ or D [28] can be as high as $0.8\left({ }^{3} \mathrm{He}\right.$ can also be used). In the case of an extracted beam, different choices for polarised targets are possible. Given the available space in the underground LHC complex, the polarised target system used by COMPASS (see [30] and reference therein) may not be optimal because of the large size of the cooling system to maintain the frozen spin mode. It seems more convenient to opt for the system of E1039 at Fermilab [11], that is a UVa-type $\mathrm{NH}_{3}$ DNP target with a strong 5T field. Since a complete comparison is presented in [31], we refrain from repeating it here. In general, $\mathrm{H}$ or D gas targets offer much better figures-of-merit with no dilution and a high polarisation. Such a comparison assumes that the data-taking periods are similar, that the effective gas pressure can be similar to that of the HERMES condition (storage cell target) and that a conventional polarised target would have a length of about one meter.

\section{Proposed measurements}

Recent simulations [32] have confirmed the very strong case for quarkonium physics [33] with AFTER @LHC. For spin physics, measurements of STSAs for single $J / \psi$ or $\Upsilon$ should also be easily accessible with the set-ups discussed above. Even with less favourable dilution factors or target transverse densities (see Fig. 1 (a)), such STSAs should tell us if the Sivers effect is suppressed or not in the gluon sector. More work remains to be done to tell down to which $P_{T}$ other states such as $\eta_{c}$ or $\chi_{c}$ could be observed. Along the lines of [13], the latter measurements are very important to set direct constraints on the gluon Sivers function, instead of on twist-3 correlators. We note that these are also interesting [34] to extract gluon TMDs of unpolarised proton, similar to the Boer-Mulders functions [35], without a polarised target.

Another very promising observable to unveil the gluon sector of the TMD is certainly the production of a pair of $J / \psi$ since both are produced colourless at low momenta [36] and it also remains a gluon-induced process even in the far backward domain. The yields for $J / \psi+J / \psi$ com- 
puted in [37] are large enough such that one can measure the corresponding STSA with sufficient precision and differential in the pair-transverse momentum which is necessary to access the $k_{T}$ of the colliding gluons. Such measurements could also be very well complemented by that of $J / \psi$ or $\Upsilon$ produced back to back to a photon [3] (see also [38] for a discussion of the interest of such a measurement with an unpolarised target) or even di-photon production, as suggested in [39], if the background can be dealt with. We therefore plan to carry out careful analyses of the projected yields and asymmetries for these reactions.

While analysing the dimuon continuum between the charmonium and bottomonium families in [32], we also noticed that the DY signal is perfectly accessible with a tractable combinatorial background which can probably be subtracted using the like-sign and/or mixed-event methods. Care should however be taken about the heavy-flavour background from charm and beauty. The asymmetries induced by these processes can nevertheless be studied separately by selecting displaced dimuons with a vertex detector. In fact, heavy-flavour STSAs are interesting on their own [40]. In view of this, we expect DY STSAs to be accessible in a very interesting kinematical region and to provide unique constraints on the quark Sivers function especially at large $x$ (large $Q$ ) where the background is by far the least problematic (see Fig. 1 (b)).

As discussed in the introduction, the studies of STSAs of isolated photons, single pions as well as jets were nicely motivated in recent works [7, 40]. For these three processes, the yields are obviously larger than for (open and hidden) heavy-flavour and DY production. Since the expected statistical samples are large, the remaining challenge could be to gain a sufficient control over the background in the measurement.

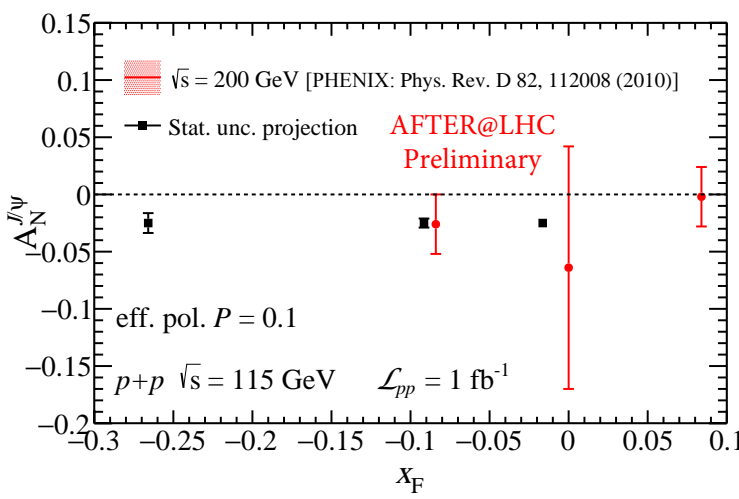

(a) $A_{N}^{J / \psi}$

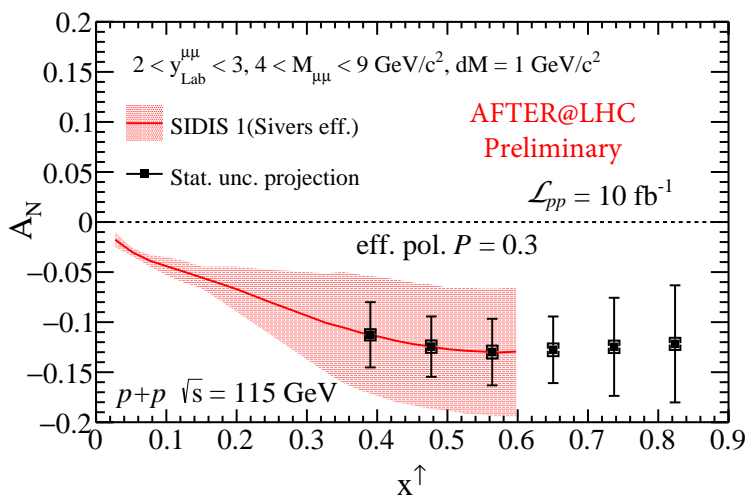

(b) $A_{N}^{\mathrm{DY}}$

Figure 1: Projected figures-of-merit for STSA of (a) $J / \psi$ and (b) DY production. The projected uncertainties are based on the indicated effective polarisations and luminosities using the simulated events of [32]. In (a), the 3 black points correspond to $2<y_{\text {Lab }}<3,3<y_{\text {Lab }}<4$ and $4<y_{\text {Lab }}<5$ centred at $A_{N}^{J / \psi}=0.025$ which is the averaged central values of the PHENIX points. In (b), the theoretical uncertainties in red are from [6]. 


\section{Conclusion}

The measurements of STSAs at a fixed-target set-up using the LHC beams with a detector covering rapidities from roughly 2 to 5 correspond to a region where asymmetries are expected to be significant. With both techniques which we discussed to study such collisions, namely an extracted beam by means of a bent crystal on a polarised target or an internal polarised gas target, the luminosity and the target polarisation characteristics will be good enough for decisive measurements both on quark and gluon sensitive probes. This naturally allows for an extended spin-physics program. In particular, the measurements which we mentioned allow for the study of the physics of initial and final state interactions which opens up an entire range of new leading-twist physics and factorisation-violating dynamics for QCD. If a system like the HERMES target is used, the performances probably exceed those of all the currently running and planned experiments.

\section{References}

[1] D. W. Sivers, Phys. Rev. D 41 (1990) 83.

[2] S. J. Brodsky, F. Fleuret, C. Hadjidakis and J. P. Lansberg, Phys. Rept. 522 (2013) 239

[3] J. P. Lansberg et al., EPJ Web Conf. 85 (2015) 02038

[4] L. Massacrier et al., arXiv:1502.00984 [nucl-ex].

[5] T. Liu and B. Q. Ma, Eur. Phys. J. C 72 (2012) 2037

[6] M. Anselmino, U. D’Alesio and S. Melis, Adv. High Energy Phys. 2015 (2015) 475040

[7] K. Kanazawa, Y. Koike, A. Metz and D. Pitonyak, Adv. High Energy Phys. 2015 (2015) 257934

[8] J. C. Collins, Phys. Lett. B 536 (2002) 43

[9] S. J. Brodsky, D. S. Hwang and I. Schmidt, Nucl. Phys. B 642 (2002) 344

[10] C. Quintans [COMPASS Collaboration], J. Phys. Conf. Ser. 295 (2011) 012163.

[11] A. Klein et al., FERMILAB-LOI-2013-01.

[12] L. D. Isenhower, et al., FERMILAB-PROPOSAL-1027.

[13] D. Boer, C. Lorcé, C. Pisano and J. Zhou, Adv. High Energy Phys. 2015 (2015) 371396

[14] A. Adare et al. [PHENIX Collaboration], Phys. Rev. D 82 (2010) 112008 [Ibid. 86 (2012) 099904]

[15] F. Yuan, Phys. Rev. D 78 (2008) 014024

[16] A. Schafer and J. Zhou, Phys. Rev. D 88 (2013) 1, 014008

[17] E. Uggerhøj, U. I. Uggerhøj, Nucl. Instrum. Meth. B 234 (2005) 31.

[18] LHC Committee, minutes of the 107th meeting, CERN/LHCC 2011-010

[19] W. Scandale, et al., CERN-SPSC-2015-039 (see section 4).

[20] G. Arduini, et al., Phys. Lett. B 422 (1998) 325.

[21] A. Asseev, et al., Phys. Rev. ST Accel. Beams 1 (1998) 022801.

[22] A. G. Afonin, et al., Phys. Rev. ST Accel. Beams 15 (2012) 081001.

[23] W. Scandale, et al., Phys. Lett. B 703 (2011) 547.

[24] K. Ehret, Nucl. Instrum. Meth. A 446 (2000) 190.

[25] A. B. Kurepin, N. S. Topilskaya and M. B. Golubeva, Phys. Atom. Nucl. 74 (2011) 446 [Yad. Fiz. 74 (2011) 467]. A. B. Kurepin and N. S. Topilskaya, Adv. High Energy Phys. 2015 (2015) 760840.

[26] M. Ferro-Luzzi, Nucl. Instrum. Meth. A 553 (2005) 388.

[27] A. Airapetian et al. [HERMES Collaboration], Nucl. Instrum. Meth. A 540 (2005) 68

[28] C. Barschel, P. Lenisa, A. Nass and E. Steffens, Adv. High Energy Phys. 2015 (2015) 463141.

[29] A. Baurichter, et al., Nucl. Instrum. Meth. B 164-165 (2000) 27.

[30] N. Doshita et al., Nucl. Instrum. Meth. A 526 (2004) 138.

[31] E. Steffens, PoS PSTP 2015 019, these proceedings.

[32] L. Massacrier, et al., Adv. High Energy Phys. 2015 (2015) 986348

[33] J. P. Lansberg, S. J. Brodsky, F. Fleuret and C. Hadjidakis, Few Body Syst. 53 (2012) 11

[34] D. Boer and C. Pisano, Phys. Rev. D 86 (2012) 094007

[35] D. Boer and P. J. Mulders, Phys. Rev. D 57 (1998) 5780

[36] J. P. Lansberg and H. S. Shao, Phys. Lett. B 751 (2015) 479

[37] J. P. Lansberg and H. S. Shao, Nucl. Phys. B 900 (2015) 273

[38] W. J. den Dunnen, J. P. Lansberg, C. Pisano and M. Schlegel, Phys. Rev. Lett. 112 (2014) 212001

[39] J. W. Qiu, M. Schlegel and W. Vogelsang, Phys. Rev. Lett. 107 (2011) 062001

[40] M. Anselmino, M. Boglione, U. D’Alesio, E. Leader and F. Murgia, Phys. Rev. D 70 (2004) 074025 